INPLASY

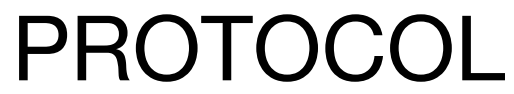

To cite: Wang et al. The benefits of Qigong exercise for fatigue symptom: a systematic review and metaanalysis. Inplasy protocol 202060009. doi:

10.37766/inplasy2020.6.0009

Received: 02 June 2020

Published: 02 June 2020

Corresponding author: Rui Wang

k444www@163.com

Author Affiliation:

Hangzhou Hospital of

Traditional Chinese Medicine

Support: Qigong Management Center

Review Stage at time of this submission: Preliminary searches.

Conflicts of interest:

The review team members declare that they have no competing interests.

\section{The benefits of Qigong exercise for fatigue symptom: a systematic review and meta-analysis}

Wang, $\mathrm{R}^{1} ; \mathrm{Wu}, \mathrm{Y}^{2} ;$ Huang, $\mathrm{X}^{3}$; Sun, $\mathrm{D}^{4}$.

Review question / Objective: What are the benefits of Qigong exercise for the improvement of fatigue symptom? We aim to generate a clinically useful summary of the interventions based on their efficacy and safety.

Condition being studied: Fatigue is a subjective feeling of patients, which is the accompanied symptom of caused by different diseases. The targeted treatments fatigue symptoms are often overlooked and have no effective cures except treatments of primary diseases. However, fatigue symptoms can't be effectively relieved, which will reduce the therapeutic effect and quality of life of patients. As a comprehensive and alternative therapy, Qigong exercise has a broad mass base in China. Its main goal is the remediation of bad physiological function, but the patient's emotions and psychology can also be changed.

INPLASY registration number: This protocol was registered with the International Platform of Registered Systematic Review and Meta-Analysis Protocols (INPLASY) on 02 June 2020 and was last updated on 02 June 2020 (registration number INPLASY202060009).

\section{INTRODUCTION}

Review question / Objective: What are the benefits of Qigong exercise for the improvement of fatigue symptom? We aim to generate a clinically useful summary of the interventions based on their efficacy and safety.
Condition being studied: Fatigue is a subjective feeling of patients, which is the accompanied symptom of caused by different diseases. The targeted treatments fatigue symptoms are often overlooked and have no effective cures except treatments of primary diseases. However, fatigue symptoms can't be effectively relieved, which will reduce the therapeutic effect 
and quality of life of patients. As a comprehensive and alternative therapy, Qigong exercise has a broad mass base in China. Its main goal is the remediation of bad physiological function, but the patient's emotions and psychology can also be changed.

\section{METHODS}

Participant or population: Patients with primary diseases-related fatigue symptoms will be included, regardless of the underlying disease, age, gender, education, ethnicity, and occupation. However, if the included literatures without providing clear diagnostic criteria have detailed description of the sufficient clinical features to existing fatigue symptoms will also be included.

Intervention: Qigong-related exercise was the main intervention (e.g. Qigong, Baduanjin, Yijinjing, Wuqinxi). The duration and frequency of therapy are not limited.

Comparator: There is no exclusion based on comparator method for this review, and the patients could be treated with any type of control group including exercise, stretching, sham Qigong, waiting list control, or other treatments.

Study designs to be included: Randomized controlled trials with no limitations on blinding or publication types will be included.

Eligibility criteria: Randomized controlled trials (RCTs) with no limitations on blinding published in English or Chinese up to June 30, 2020 will be included. Patients with primary diseases-related fatigue symptoms will be included, regardless of the underlying disease, age, gender, education, ethnicity, and occupation. However, if the inclusion patients in the literature exist in series complications, mental illness, cognitive communication influences, or pregnant, will be excluded. Qigong- related exercises alone (any forms of Qigong category, e.g. Baduanjin, Yijinjing, and Wuqinxi) or Qigong combined with any other basic treatments was the main intervention in the observation group. There is no limit to the duration and frequency of therapy. There is no exclusion based on comparator method for this review, and the patients could be treated with any type of control group including exercise, stretching, sham Qigong, waiting list control, or other treatments.

Information sources: The following databases will be searched: PubMed, Web of Science, Embase, Cochrane Library, China Biology Medicine disc, China National Knowledge Infrastructure, Wanfang and VIP Data Knowledge Service Platform. Clinical trial registries are part of other resource searches, like ClinicalTrials.gov will be searched for ongoing trials with unpublished data. Furthermore, the reference lists of included studies and previous relevant reviews will be manually retrieved to further locate additional trials to identify additional articles that met our inclusion criteria. The potential conference proceedings will be evaluated; if necessary we can get in touch with the trial authors to obtain complete data.

Main outcome(s): (1) Flinders Fatigue Scale (FSS); (2) Functional Assessment of Chronic Illness Therapy-Fatigue (FACIT-F); (3) Visual Analogue Scale-Fatigue (VAS-F); (4) Multidimensional Fatigue Inventory (MFI).

Additional outcome(s): (1) Brief Fatigue Inventory (BFI); (2) Fatigue Symptom Inventory (FSI); (3) Quality of life.

Quality assessment / Risk of bias analysis: Two reviewers will independently assess the quality of the selected studies according to the Cochrane Collaboration's tool for randomized controlled trials. Items will be evaluated in three categories: Low risk of bias, unclear bias and high risk of bias. The following characteristics will be evaluated: Random sequence generation (selection bias), Allocation concealment (selection bias), Blinding of participants and personnel (performance bias), Incomplete outcome data (attrition bias), Selective reporting (reporting bias), other 
biases results from these questions will be graphed and assessed using Review Manager 5.3.

Strategy of data synthesis: Risk ratio (RR) for both fixed and random effects models (weighting by inverse of variance) will be used. A continuity correction will also be used for cells with zero values. Betweenstudy heterogeneity will be assessed using the ?2 (Cochran Q) and 12 statistics. According to the Cochrane handbook, the 12 will be considered non-important $(60 \%)$. Results will be assessed using forest plots and presented as RRs for the main outcome and secondary outcomes. An influence analysis will be performed to ascertain the results of the meta-analysis by excluding each of the individual studies. Publication bias will be assessed by a funnel plot for meta-analysis and quantified by the Egger method. Statistical analysis will be conducted using STATA software for Mac v15.0 (STATA Corp College Station, Texas) [module meta"] and $R$ studio v1.0.136 (The R Foundation for Statistical Computing) [package "meta v4.2"].

Subgroup analysis: If sufficient data allow, we plan to perform analysis on the following subgroups of interest: (1)Primary diseases; (2)Qigong exercise type; (3)Control intervention type; (4)Follow-up period. We will assess subgroup effects for interpreting the heterogeneity.

Sensibility analysis: If there are sufficient data which available to analyze, we will conduct sensitivity analysis on the primary outcomes to test the robustness of the review conclusions, including the quality of the methods, the quality of the studies, and the impact of sample size and missing data.

Language: English or Chinese-language.

Country(ies) involved: China.

Keywords: Fatigue; Qigong; Humans.

Contributions of each author:

Author 1 - Rui Wang - Author 1 was in charge of conceptualization and writing- original draft of the manuscript. And this author contributed to the development of the selection criteria, and the risk of bias assessment strategy.

Author 2 - Yeqi Wu - Author 2 was in charge of data search, analysis, and statistics of the manuscript.

Author 3 - Xueyan Huang - Author 3 was in charge of data search, analysis, and statistics of the manuscript. And this author contributed to the development of the selection criteria, and the risk of bias assessment strategy.

Author 4 - Dai Sun - Author 4 read, provided feedback and approved the final manuscript. 